\title{
The Relationship Between Timing of Pre-Transplant Kidney Biopsy, Graft Loss, and Survival in Kidney Transplantation: An Italian Cohort Study.
}

\section{Federica Odaldi}

IRCCS, Azienda Ospedaliero-Universitaria di Bologna

Matteo Serenari

IRCCS, Azienda Ospedaliero-Universitaria di Bologna

Giorgia Comai

IRCCS, Azienda Ospedaliero-Universitaria di Bologna

\section{Gaetano La Manna}

IRCCS, Azienda Ospedaliero-Universitaria di Bologna

\section{Raffaele Bova}

IRCCS, Azienda Ospedaliero-Universitaria di Bologna

Giacomo Frascaroli

IRCCS, Azienda Ospedaliero-Universitaria di Bologna

\section{Deborah Malvi}

IRCCS, Azienda Ospedaliero-Universitaria di Bologna

\section{Lorenzo Maroni}

IRCCS, Azienda Ospedaliero-Universitaria di Bologna

Francesco Vasuri

IRCCS, Azienda Ospedaliero-Universitaria di Bologna

Giuliana Germinario

IRCCS, Azienda Ospedaliero-Universitaria di Bologna

Olga Baraldi

IRCCS, Azienda Ospedaliero-Universitaria di Bologna

Irene Capelli

IRCCS, Azienda Ospedaliero-Universitaria di Bologna

\section{Vania Cuna}

IRCCS, Azienda Ospedaliero-Universitaria di Bologna

\section{Gabriela Sangiorgi}

IRCCS, Azienda Ospedaliero-Universitaria di Bologna

Antonietta D'Errico

IRCCS, Azienda Ospedaliero-Universitaria di Bologna

Massimo Del Gaudio 
IRCCS, Azienda Ospedaliero-Universitaria di Bologna

\section{Valentina Bertuzzo}

IRCCS, Azienda Ospedaliero-Universitaria di Bologna

\section{Chiara Zanfi}

IRCCS, Azienda Ospedaliero-Universitaria di Bologna

\section{Maurizio Sessa}

University of Copenhagen

Matteo Ravaioli ( $\square$ mrava1@hotmail.com )

IRCCS, Azienda Ospedaliero-Universitaria di Bologna

\section{Research Article}

Keywords: solid tumors, kidney clinical, outcome, kidney biopsy, graft survival

Posted Date: December 31st, 2020

DOI: https://doi.org/10.21203/rs.3.rs-130924/v1

License: (9) This work is licensed under a Creative Commons Attribution 4.0 International License. Read Full License 


\section{Abstract}

Kidney biopsy is performed to assess if an extended criteria graft can be used for transplantation. It may be performed before or after cross-clamping during organ procurement. This study aims to evaluate whether the timing of biopsy may modify cold ischemia times (CIT) and/or graft outcomes. Kidney transplants performed in our centre from January 2007 to December 2017 were analysed. Grafts with preimplantation kidney biopsy were included. Biopsies were performed during surgical back table (ESKB, exsitu kidney biopsy) until 2012 and since then, before the aortic cross-clamping (ISKB, in situ kidney biopsy). To overcome biases owing to different distribution, a propensity score model was developed. The study population consists in 322 patients, 115 ESKB and 207 ISKB. Cold ischemia time was significantly lower for ISKB (730 min ISKB vs. 840 min ESKB, p-value = 0.001). In both crude (OR 0.27; $95 \%$ Confidence Interval, $95 \% \mathrm{Cl} 0.12-0.60 ; \mathrm{p}$-value $=0.002)$ and adjusted analyses $(\mathrm{OR} 0.37 ; 95 \% \mathrm{Cl}$ 0.14-0.94; p-value =0.039), ISKB was associated with a reduced odd of graft loss when compared to ESKB. Performing pre-implantation kidney biopsy during the recovery, prior to the aortic cross-clamping, may be a strategy to reduce cold ischemia time and improve transplant outcomes.

\section{Introduction}

The shortage of organ donors is still a major concern in kidney transplantation. Older donors and/or donors with multiple comorbidities- the so called extended criteria donors- represent a main organ resource with an acceptable but reduced graft survival, despite a higher incidence of delayed graft function.[1, 2] The process for an optimal selection and allocation of kidney grafts includes many factors such as recipient characteristics, donor characteristics and immunological compatibility between them. In many countries, including Italy, a kidney biopsy is usually obtained at the end of organ procurement to check any chronic changes and/or pathological deterioration with the final aim of assessing whether a marginal graft can be considered for transplantation or has to be discarded.[3, 4] Moreover, a kidney biopsy can be used to decide whether to perform a single (SKT) or a dual kidney transplantation (DKT). [4-7] Performing a pre-implantation kidney biopsy may lead to increased cold ischemia times (CIT), due to the histological examination which is time-consuming. However, a possible strategy to reduce the CIT could be to perform a procurement biopsy before the aortic cross-clamping of the donor, namely in situ kidney biopsy (ISKB), sending the biopsy immediately to the pathologist. From 2012, we have started to perform ISKB in order to see its effect on CIT and graft outcomes.

The primary aim of this study was to compare the survival and graft loss among patients receiving ISKB versus those receiving biopsies performed after cross-clamping, namely ex-situ kidney biopsy (ESKB).

\section{Materials And Methods}

\subsection{Study population and cohorts}


All consecutive kidney transplants performed in our Institution between January 2007 and December 2017 performed at our hospital were evaluated. The study population included kidneys from donors after brain death to which an allocation biopsy was made. All kidneys were from extended criteria donors (ECD) defined as donors with age $\geq 60$ years, or age between 50 and 59 years with at least one of the following: arterial hypertension, serum creatinine $\geq 1.5 \mathrm{mg} / \mathrm{dl}$, or cardiovascular accident death. In the study population were included only kidneys have been transplants from donors within our region (e.g., Emilia Romagna, Italy), where the distance from the pathologist and site of procurement was less than $150 \mathrm{~km}$. The organs are allocated in the region based on: ABO compatibility, mismatch HLA, PRA score, age of dialysis, donor-recipient age match, complex clinical conditions, risk match (infectious/ neoplastic) donor/recipient, transplant type (kidney-pancreas, double kidney, single kidney). The study population was divided in two cohorts or rather patients that received biopsy during surgical back table, at the end of the procurement procedure (i.e., ESKB) and those for which biopsies were performed before the aortic cross-clamping at the beginning of the procurement procedure (i.e., ISKB). Informed consent was obtained from all subjects or participants.

\subsection{Kidney biopsy}

Before 2012 (2007-2012), biopsies were performed as EKSB, whereas, between 2012 and the end of the study (August 2020) biopsies were performed as ISKB. All biopsies were wedge biopsies performed with a skin punch. The biopsy was always performed in the inferior pole of the kidney and closed with a pledget suture. The histological sections were embedded in Serra liquid (formalin, alcohol, glacial acid acetic) and immediately sent to experienced renal pathologist available in Bologna Transplant Center 24 hours a day, it was subjected to microwave processing and Karpinski's score is then performed. Karpinsky score evaluates the presence of glomerulosclerosis, -tubular damage, interstitial fibrosis, arterial sclerosis and arteriolar narrowing,[8] grading all these histological findings from 0 to 12 : kidneys with score $0-3$ were allocated for SKT, with score 4- 6 for DKT, whereas kidneys with score $>6$ were discarded.[4]

\subsection{Data collection}

Donor and recipient's features collected during the study period included demographical data, comorbidities, kidney donor profile index (KDPI), Karpinsky score, and recipient's aetiology of kidney disease. Kidney Donor Risk Index (KDRI) represents the relative risk of post-transplant graft failure from a particular deceased donor compared to the average donor. The KDPI is a remapping of the kidney donor risk index into a cumulative percentage scale. The KDPI was calculated evaluating the presence of the following donor comorbidities: hypertension, diabetes, cardiopathy, vascular disease, dislipidemic disease, tabagism, nephropathy, chronic use of medications, serum creatinine, anti HCV positivity and cerebrovascular death.[9] A donor with a KDPI of $80 \%$ has a higher expected risk of graft failure than $80 \%$ of all kidney donors recovered last year.[9] Renal grafts were classified according to Karpinski score.[4] DGF was defined as the reported need for two dialysis within 1-week post- transplantation.[10-12]

\subsection{Follow-up period}


Patients were followed from the date of the kidney transplantation (e.g., index date) to the occurrence of the study outcome, or at the end of the study period (e.g., 01/08/2020).

\subsection{Study outcomes}

The study outcomes were overall survival and graft loss during the follow-up period.

\subsection{Statistical analysis}

At the index date, demographic and clinical characteristics described in 2.3 were compared between the two cohorts. Mann-Whitney test was used to compare continuous variables whereas Chi-squared test or Fisher's exact test was used to compare categorical variables. A logistic regression model was used to compute the propensity score of being included in the cohort ESKB versus ISKB based study variables described in 2.3. Propensity score analysis has been found to be superior to standard adjustment techniques in clinical epidemiology when dealing with a large number of covariates likes in the setting of this study.[13] In the crude analyses, only variables exposure and the outcome were included in the logistic regression model to compute the odds ratio (OR) for the study outcome. In the adjusted analyses, the propensity score was added to the aforementioned logistic regression model. Competing risk was assessed by looking at differential censoring patterns of the two cohorts over the follow-up time. Survival probability curves were estimated using the Kaplan-Meier method for all-causes mortality and the cumulative incidence curve for graft loss. The comparison between survival curves for all-causes mortality was performed using the log-rank test while the comparison of cumulative incidence functions was compared using the Gray test. To identify clinically relevant subgroups at higher risk of the study outcome, we used the conditional inference tree (i.e., machine learning technique). The mathematical theory, validity, and usefulness of conditional inference tree for subgroup analysis in clinical epidemiology has been described elsewhere.[14-19] Data analysis and management was performed using R (version 3.5.0, R Development Core Team).

\subsection{Ethical considerations}

The study was approved by the local institutional review committee, Comitato Etico Indipendente di Area Vasta Emilia Centro (CE-AVEC) (133/2015/U/Sper).

\section{Results}

In the study period, 746 kidney transplants (KT) from deceased brain death donors were performed in our transplant center. Among them, a pre-implantation kidney biopsy was performed in 331 cases (44.4\%). Nine cases were excluded due to nonlocal donations (Fig. 1).

According to the inclusion criteria, the final population consisted of $322 \mathrm{KT}$ with a pre-implantation biopsy. 115 out of 322 (35.7\%) biopsies were performed ex-situ (ESKB) whereas 207 (64.2\%) in situ before the aortic cross-clamping (ISKB). The median recipient age was significantly higher in the ESKB cohort compared to ISKB (71 vs 64 years) whereas diabetes was more prevalent in the ISKB cohort. The median CIT was significantly lower in the ISKB group compared to ESKB (730 min INKB vs. 840 min 
ESKB, p-value $=0.000$ ). No organs were discarded for surgical damage performed during ISKB. In four cases it was necessary to re-perform the biopsy due to the insufficiency or inadequacy of the material taken with the ISKB.

Baseline characteristics of the two cohorts are shown in Table 1. 
Table 1

Baseline characteristics of the study population.

\begin{tabular}{|c|c|c|c|c|c|}
\hline Variable & Level & $\begin{array}{l}\text { ESKB }(n= \\
115)\end{array}$ & $\begin{array}{l}\text { ISKB } \\
(n=207)\end{array}$ & $\begin{array}{l}\text { Total }(n= \\
322)\end{array}$ & $\begin{array}{l}\mathrm{p}- \\
\text { value }\end{array}$ \\
\hline \multicolumn{6}{|l|}{ Receiver } \\
\hline Age & median (range) & $71(53,82)$ & $64(27,83)$ & $66(27,83)$ & $\begin{array}{l}< \\
0.001\end{array}$ \\
\hline \multirow[t]{2}{*}{ Sex } & Female & $48(41.7)$ & $70(33.8)$ & $118(36.6)$ & \\
\hline & Male & 67 (58.3) & $137(66.2)$ & $204(63.4)$ & 0.195 \\
\hline \multirow{11}{*}{$\begin{array}{l}\text { Year of inclusion in } \\
\text { the cohort }\end{array}$} & 2007 & $26(22.6)$ & $0(0.0)$ & $26(8.1)$ & \\
\hline & 2008 & $22(19.1)$ & $0(0.0)$ & $22(6.8)$ & \\
\hline & 2009 & $28(24.3)$ & $0(0.0)$ & $28(8.7)$ & \\
\hline & 2010 & $22(19.1)$ & $0(0.0)$ & $22(6.8)$ & \\
\hline & 2011 & $17(14.8)$ & $0(0.0)$ & $17(5.3)$ & \\
\hline & 2012 & $0(0.0)$ & $33(15.9)$ & $33(10.2)$ & \\
\hline & 2013 & $0(0.0)$ & $23(11.1)$ & $23(7.1)$ & \\
\hline & 2014 & $0(0.0)$ & $23(11.1)$ & $23(7.1)$ & \\
\hline & 2015 & $0(0.0)$ & $36(17.4)$ & $36(11.2)$ & \\
\hline & 2016 & $0(0.0)$ & 39 (18.8) & $39(12.1)$ & \\
\hline & 2017 & $0(0.0)$ & 53 (25.6) & $53(16.5)$ & $\begin{array}{l}< \\
0.001\end{array}$ \\
\hline Body Mass Index & median (range) & $\begin{array}{l}\text { 24.4 (17.3, } \\
33.1)\end{array}$ & $\begin{array}{l}25(15.6 \\
33.2)\end{array}$ & $\begin{array}{l}\text { 24.8 (15.6, } \\
33.2)\end{array}$ & 0.615 \\
\hline \multirow[t]{4}{*}{ ABO group } & 0 & 59 (51.3) & $85(41.1)$ & $144(44.7)$ & \\
\hline & A & 45 (39.1) & $93(44.9)$ & $138(42.9)$ & \\
\hline & $A B$ & $3(2.6)$ & $8(3.9)$ & $11(3.4)$ & \\
\hline & B & $8(7.0)$ & $21(10.1)$ & $29(9.0)$ & 0.322 \\
\hline \multirow[t]{3}{*}{ Diagnosis } & Berger's disease & $14(12.2)$ & $10(4.8)$ & $24(7.5)$ & \\
\hline & $\begin{array}{l}\text { Chronic } \\
\text { glomerulonephritis }\end{array}$ & $7(6.1)$ & $23(11.1)$ & $30(9.3)$ & \\
\hline & $\begin{array}{l}\text { Chronic } \\
\text { glomerulosclerosis }\end{array}$ & $29(25.2)$ & $45(21.7)$ & $74(23.0)$ & \\
\hline
\end{tabular}




\begin{tabular}{|c|c|c|c|c|c|}
\hline Variable & Level & $\begin{array}{l}\text { ESKB }(n= \\
115)\end{array}$ & $\begin{array}{l}\text { ISKB } \\
(n=207)\end{array}$ & $\begin{array}{l}\text { Total }(n= \\
322)\end{array}$ & $\begin{array}{l}\mathrm{p} \text { - } \\
\text { value }\end{array}$ \\
\hline & $\begin{array}{l}\text { Diabetic } \\
\text { nephropathy }\end{array}$ & $1(0.9)$ & $9(4.3)$ & $10(3.1)$ & \\
\hline & $\begin{array}{l}\text { Hypertensive } \\
\text { nephropathy }\end{array}$ & $15(13.0)$ & $34(16.4)$ & $49(15.2)$ & \\
\hline & Interstitial nephritis & $8(7.0)$ & $4(1.9)$ & $12(3.7)$ & \\
\hline & Others & $11(9.6)$ & 35 (16.9) & $46(14.3)$ & \\
\hline & Renal polycystosis & $30(26.1)$ & $47(22.7)$ & $77(23.9)$ & 0.007 \\
\hline \multirow{3}{*}{$\begin{array}{l}\text { Number of } \\
\text { transplants }\end{array}$} & 1 & $115(100.0)$ & 189 (91.3) & $304(94.4)$ & \\
\hline & 2 & $0(0.0)$ & $16(7.7)$ & $16(5.0)$ & \\
\hline & 3 & $0(0.0)$ & $2(1.0)$ & $2(0.6)$ & 0.005 \\
\hline Diabetes mellitus & Yes & $1(0.9)$ & $26(12.6)$ & $27(8.4)$ & $\begin{array}{l}< \\
0.001\end{array}$ \\
\hline $\begin{array}{l}\text { Cardiovascular } \\
\text { disorders }\end{array}$ & Yes & $88(76.5)$ & $153(73.9)$ & $241(74.8)$ & 0.701 \\
\hline Hypotension & Yes & $2(1.7)$ & $1(0.5)$ & $3(0.9)$ & 0.603 \\
\hline Cirrhosis & Yes & $1(0.9)$ & $11(5.3)$ & $12(3.7)$ & 0.087 \\
\hline \multicolumn{6}{|l|}{ Donor } \\
\hline Age & median (range) & $69(46,82)$ & $70(34,85)$ & $69(34,85)$ & 0.707 \\
\hline \multirow[t]{2}{*}{ Ethnicity } & Caucasian & $115(100.0)$ & 205 (99.0) & $320(99.4)$ & \\
\hline & Non Caucasian & $0(0.0)$ & $2(1.0)$ & $2(0.6)$ & 0.751 \\
\hline \multirow[t]{2}{*}{ Sex } & Female & $52(45.2)$ & 105 (50.7) & $157(48.8)$ & \\
\hline & Male & $63(54.8)$ & $102(49.3)$ & $165(51.2)$ & 0.405 \\
\hline Body Mass Index & median (range) & $\begin{array}{l}25.7(19.5, \\
35.4)\end{array}$ & $\begin{array}{l}25.7(16.2, \\
40.4)\end{array}$ & $\begin{array}{l}25.7(16.2 \\
40.4)\end{array}$ & 0.904 \\
\hline \multirow[t]{4}{*}{ AB0 group } & 0 & $59(51.3)$ & $90(43.5)$ & $149(46.3)$ & \\
\hline & $A$ & $45(39.1)$ & $93(44.9)$ & $138(42.9)$ & \\
\hline & $A B$ & $3(2.6)$ & $4(1.9)$ & $7(2.2)$ & \\
\hline & B & $8(7.0)$ & $20(9.7)$ & $28(8.7)$ & 0.505 \\
\hline
\end{tabular}




\begin{tabular}{|llllll|}
\hline Variable & Level & $\begin{array}{l}\text { ESKB }(\mathbf{n}= \\
\mathbf{1 1 5})\end{array}$ & $\begin{array}{l}\text { ISKB } \\
(\mathbf{n = 2 0 7 )}\end{array}$ & $\begin{array}{l}\text { Total }(\mathbf{n}= \\
\mathbf{3 2 2})\end{array}$ & $\begin{array}{l}\mathbf{p}- \\
\text { value }\end{array}$ \\
\hline $\begin{array}{l}\text { Cardiovascular } \\
\text { disorders }\end{array}$ & Yes & $29(25.2)$ & $77(37.2)$ & $106(32.9)$ & 0.038 \\
\hline Vasculopathy & Yes & $8(16.7)$ & $35(17.0)$ & $43(16.9)$ & 1.000 \\
\hline Dyslipidemia & Yes & $21(18.3)$ & $59(28.6)$ & $80(24.9)$ & 0.053 \\
\hline Tabagism & Yes & $30(26.1)$ & $68(33.0)$ & $98(30.5)$ & 0.244 \\
\hline Nephropathy & Yes & $3(2.6)$ & $1(0.5)$ & $4(1.2)$ & 0.256 \\
\hline Hypertension & Yes & $69(60.5)$ & $140(67.6)$ & $209(65.1)$ & 0.247 \\
\hline Diabetes mellitus & Yes & $11(9.6)$ & $39(18.8)$ & $50(15.5)$ & 0.041 \\
\hline HCV & Yes & $1(0.9)$ & $2(1.0)$ & $3(0.9)$ & 1.000 \\
\hline KDPI & median (range) & $95(42$, & $95(30$, & $95(30$, & 0.776 \\
\hline Outcomes & $100)$ & $100)$ & $100)$ & \\
\hline CIT & & & & & 0.000 \\
\hline DGF & median (range) & $840(720$, & $730(635$, & $780(660$, & $900)$ \\
\hline
\end{tabular}

\subsection{Graft lost}

In total, 28 patients (8.4\%) out of 332 included in the study population had graft loss of which, 10 (4.8\%) among those in the ISKB cohort and 18 (15.6\%) in the ESKB cohort. In both crude (OR 0.27; 95\% $\mathrm{Cl} 0.12-$ $0.60 ;$-value $=0.002)$ and adjusted analyses (OR 0.37; 95\% Cl 0.14-0.94; p-value $=0.039)$, ISKB was associated with a reduced odd of graft loss when compared to ESKB. Accordingly, the Gray test ( $p$-value $=0.005$ ) indicated a statistically significant difference in cumulative incidence functions between the two cohorts with an increased risk of graft loss for patients receiving ESKB (Fig. 2). In subgroup analyses, the conditional inference tree identified patients with delayed graft function as the subgroup with the highest risk of graft loss in the ISKB cohort $(p=0.003)$ (Fig. 3).

\subsection{Overall survival}

Among patients included in the study population, 18 (5.6\%) died of which, $4(1.9 \%)$ among those in the ISKB cohort and $14(12.2 \%)$ in the ESKB cohort. In the crude analyses, ISKB was associated with a reduced odd of death when compared to ESKB (OR 0.14; 95\%Cl0.04-0.41; p-value < 0.001 ). Accordingly, the log-rank test $(p$-value $=0.0014)$ indicated a statistically significant difference in overall survival between the two cohorts with an increased survival benefit for patients receiving ISKB (Fig. 4). Also in the adjusted analyses, ISKB was associated with a reduced odd of death when compared to ESKB (OR 0.25; 
$95 \% \mathrm{Cl} 0.06-0.85 ; \mathrm{p}$-value $=0.035)$. No subgroups with statistically significant predictors of death were identified for the ISKB cohort.

\section{Discussion}

This study shows that a pre-implantation kidney biopsy performed during organ procurement, before the aortic cross-clamping significantly reduced mortality and graft loss when compared to ex-situ kidney biopsy.

The Italian allocation policy provides to perform a pre-implantation biopsy to accept or decline ECD kidneys for transplantation and whether to decide if SKT or DKT should be performed. ${ }^{4}$ In other countries, performing pre-implantation biopsy is still debated and controversial. For example, the United Network of Organ Sharing (UNOS) Kidney Transplantation Committee has recently approved a new allocation policy which is based on the KDPI and not on the histological features. ${ }^{9}$ Even for this context, where the allocation policy does not include a pre-transplant biopsy performed routinely, some authors suggests that for hight KDPI values (even for KDPI close to 100), a pre-transplant kidney biopsy could be important to support the suitability of the graft and the allocation to SKT or DKT.[20, 21] Donors included in this study had a very high median KDPI (95\%) and, despite this, we maintained the survival of the graft and the patient comparable to the transplant performed with donors with standard criteria.[22]

One of the main concerns of allocation systems using pre-implantation kidney biopsy, is that the histological examination is time consuming and may increase CIT. Usually, the biopsy is performed at the end of procurement, on surgical back table. From 2012 we have started to perform kidney biopsies as one of the first procedures during multiorgan procurement, sending them immediately to the pathologist while the recovery is still ongoing. Our hypothesis was that this may have led to an improved outcome due to a decrease of CIT. In our study, the median CIT was lower in transplants with ISKB than those with ESKB. Since the cascade of events leading to kidney injury is initiated by cold ischemia, it is reasonable to think that the longer is the duration of cold ischemia, the higher is its harmful impact [23] and it is plausible that a prolonged CIT may have a negative impact on graft survival: the injuries consequent to the cold storage can lead to kidney damage and to the activation of the immune response, both of which may affect the outcome after transplantation.[13] In literature, there is no consensus on the precise relationship of CIT with the transplantation outcomes. The injuries consequent to the cold storage can lead to kidney damage and to the activation of the immune response, both of which affect the outcome after transplantation. Debout et al.[24] reported that even short differences in CIT may influence both graft and patient survival. A recent Dutch study reported that a higher CIT is associated with an increased risk of graft failure.[17] Our study confirms these hypotheses, since overall and graft survival was significantly higher in patients with ISKB compared to those with ESKB.

Our study has some limitations. First, performing the ISKB is less useful when kidney biopsies are not performed routinely to allocate grafts. In certain contexts, furthermore, this method is not applicable because of a different organization and the time required for the pathological examination. In our Region, 
the allocation of the biopsy involves a complex organization as all biopsies are centralized in our center and the availability of experienced pathologist 24 hours a day is necessary. This does not change our standard of practice where the same specialist has to be available also to analyse the liver biopsy.[25, 26] We have selected only donors within our Region whose donation centers were at most $150 \mathrm{~km}$ far from the pathological anatomy center, thus not representing a bias when CIT was measured. This technique becomes more difficult to apply in contexts where the allocation is on larger territory. The allocation system in Italy provides national distribution only for special programs (pediatric list, emergencies, hyperimmune patients), while in other cases, also for organs from marginal donors, the allocation is regional.

Secondly, there could be the risk of not having a diagnostic biopsy when performing the ISKB. However, in our series, only in 4 cases we needed to repeat the kidney biopsies on back table as we did not have enough tissue for the sampling analysis. More importantly, no kidneys were lost for a surgical damage. The same surgical team performed the sampling of liver and kidneys. This allowed for more experienced surgeons and contributed to have few complications. Nevertheless, ISKB must be performed with care and attention in order to avoid any possible parenchymal bleeding or hematoma.

Furthermore, being a retrospective study, the two populations were different, despite the propensity score analysis should help to limit this gap. A randomized study would be ideal at this point, but our positive results do not allow us to go back from such an effective strategy. This procedure is easily executable in centres where the same surgical team provides liver and kidneys also during multiorgan procurement and could be easily widespread.

Finally, it should be mentioned that all patients underwent to ESKB were enrolled in the period 2008-2011 while those underwent ISKB in the period 2012-2017. Consequently, we were not able to adjust our estimates for the year of enrolment in the study population.

Performing pre-implantation kidney biopsy during the recovery, prior to the aortic cross-clamping, may be a strategy to reduce cold ischemia time and improve transplant outcomes.

\section{Abbreviations}

$\mathrm{BMI}$, body mass index

$\mathrm{Cl}$, confidence interval

CIT, cold ischemia time

DGF, delayed graft function

DKT, dual kidney transplantation

ESKB, ex-situ kidney biopsy 
HLA, human leukocyte antigens

ISKB, in-situ kidney biopsy

KDPI, kidney donor profile index

$\mathrm{KDRI}$, kidney donor risk index

$\mathrm{KT}$, kidney transplant

$\mathrm{OR}$, odds ratio

PS, propensity score

PRA, panel reactive antibody

SKT, single kidney transplantation

UNOS, United Network for Organ Sharing

\section{Declarations}

\section{Conflict of Interest Statement}

The authors declare no conflicts of interest.

\section{Funding}

The authors received no specific funding for this work.

\section{Data Availability Statement}

Raw data were generated at IRCCS, Azienda Ospedaliero-Universitaria di Bologna. Derived data supporting the findings of this study are available from the corresponding author (MR) on request.

\section{Author Contributions Statement}

Conceptualization: M.R., F.O., G.C., G.L.M., A.D., M.D.G.; Literature search: R.B., G.F., D.M., L.M., F.V., M.S.; Acquisition of data: G.G., O.B., I.C., V.C., G.S., V.R.B., C.Z.; Statistical analysis: M.S.

Writing/Reviewing/Editing: F.O., M.S., G.C., G.L.M., R.B., G.F., D.M., L.M., F.V., G.G., O.B., I.C., V.C., G.S., A.D., M.D.G., V.R.B., C.Z., M.S., M.R.

\section{Methods Statement}

All methods were carried out in accordance with relevant guidelines and regulations.

\section{Protocols Statement}


All experimental protocols were approved by the local institutional committee: 'Comitato Etico Indipendente di Area Vasta Emilia Centro (CE-AVEC)'.

\section{References}

1. Florman, S. et al. Efficacy and Safety Outcomes of Extended Criteria Donor Kidneys by Subtype: Subgroup Analysis of BENEFIT- EXT at 7 Years After Transplant. Am. J. Transplant. 17, 180-190 (2017).

2. Metzger, R. A. et al. Expanded Criteria Donors for kidney transplantation. Am. J. Transplant. 3, 114125 (2003).

3. Matas, A. J. et al. Annual Data Report: Kidney. Am. J. Transplant. 13, 11-46 (2013).

4. Remuzzi, G. et al. Early experience with dual kidney transplantation in adults using expanded donor criteria. Double Kidney Transplant Group (DKG). J. Am. Soc. Nephrol. 10, 2591-2598 (1999).

5. Pierobon, E. S. et al. Optimizing utilization of kidneys from deceased donors over 60 years:Five years outcomes after implantation of a combined clinical and histological allocation algorithm. Transpl. Int. 26, 833-841 (2013).

6. Fernández-Lorente, L. et al. Long term results of biopsy-guided selection and allocation of kidneys from older donors in older recipients. Am. J. Transplant. 12, 2781-2788 (2012).

7. Stratta, R. J. et al. Dual kidney transplants from adult marginal donors successfully expand the limited deceased donor organ pool. Clin. Transpl. 30, 380-392 (2016).

8. Karpinski, J. et al. Outcome of kidney transplantation from hight risk donors is determined by both structure and function. Transplantation. 67, 1162-1167 (1999).

9. Tso, P. L. Access to renal transplantation for the elderly in the face of new allocation policy. A review of contemporary perspectives on "older" issues. Transplant. Rev. 28, 6-14 (2014).

10. Irish, W. D. et al. Nomogram for predicting the likelihood of delayed graft function in adult deceased renal transplant recipients. J. Am. Soc. Nephrol. 14, 2967-2974 (2003).

11. Ojo, A. O., Wolfe, R. A., Held, P. J., Port, F. K. \& Schmouder, R. L. Delayed graft function: risk factors and implications for renal allograft survival. Transplantation. 63, 968-974 (1997).

12. Perico, N., Cattaneo, D., Sayegh, M. H. \& Remuzzi, G. Delayed graft function in kidney transplantation. The Lancet. 364, 1814-1827 (2004).

13. Patorno, E., Glynn, R. J., Hernández-Díaz, S., Liu, J. \& Schneeweiss, S. Studies with many covariates and few outcomes: selecting covariates and implementing propensity-score-based confounding adjustments. Epidemiology. 25, 268-278 (2014).

14. Tsai, W. M., Zhang, H., Buta, E., O'Malley, S. \& Gueorguieva, R. A modified classification tree method for personalized medicine decisions. Stat Interface. 9, 239-253 (2016).

15. Sessa, M. et al. Metoprolol versus Carvedilol in Patients with Heart Failure, Chronic Obstructive Pulmonary Disease, Diabetes Mellitus, and Renal Failure. Am. J. Cardiol. 125, 1069-1076 (2020). 
16. Sessa, M., Mascolo, A., Dalhoff, K. P. \& Andersen, M. The risk of fractures, acute myocardial infarction, atrial fibrillation and ventricular arrhythmia in geriatric patients exposed to promethazine. Expert Opin Drug Saf. 19, 349-357 (2020).

17. Sessa, M. et al. Relationship between heart failure, concurrent chronic obstructive pulmonary disease and beta-blocker use: a Danish nationwide cohort study. Eur. J. Heart. Fail. 20, 548- 556 (2018).

18. Sessa, M. et al. Effect of Chronic Kidney Diseases on Mortality among Digoxin Users Treated for Non-Valvular Atrial Fibrillation: A Nationwide Register-Based Retrospective Cohort Study. PLoS One. 11, e0160337 (2016).

19. Hashimi, H., Andersen, M. \& Sessa, M. Predictors of quetiapine extended-release formulation add-on in older patients exposed to antidepressant drugs: A Danish register-based cohort study. Int J Geriatr Psychiatry. May 19, https://doi.org/10.1002/gps.5351 (2020).

20. Gandolfini, I. et al. The Kidney Donor Profile Index (KDPI) of marginal donors allocated by standardized pretransplant donor biopsy assessment: distribution and association with graft outcomes. Am. J. Transplant. 14, 2515-2525 (2014).

21. Ekser, B., Powelson, J. A., Fridell, J. A., Goggins, W. C. \& Taber, T. E. Is the kidney donor profile index (KDPI) universal or UNOS-specific?. Am. J. Transplant. 18, 1031-1032 (2018).

22. El-Husseini, A. et al. Outcome of kidney transplant in primary, repeat, and kidney-after-nonrenal solidorgan transplantation: 15-year analysis of recent UNOS database. Clin. Transplant. https://doi.org/10.1111/ctr.13108 (2017).

23. Ponticelli, C. The impact of cold ischemia time on renal transplant outcome. Kidney Int. 87, 272-275 (2015).

24. Pérez Valdivia, M. A. et al. Impact of cold ischemia time on initial graft function and survival rates un renal transplants from deceased donors performed in Adalusia. Transplant. Proc. 43, 2174-6 (2011).

25. Ravaioli, M. et al. Liver transplantations with donors aged 60 years and above: the low liver damage strategy. Transpl. Int. 22, 423-433 (2009).

26. Ravaioli, M. et al. Risk avoidance and liver transplantation: a single-center experience in a National Network. Ann. Surg. 264, 778-786 (2016).

\section{Figures}




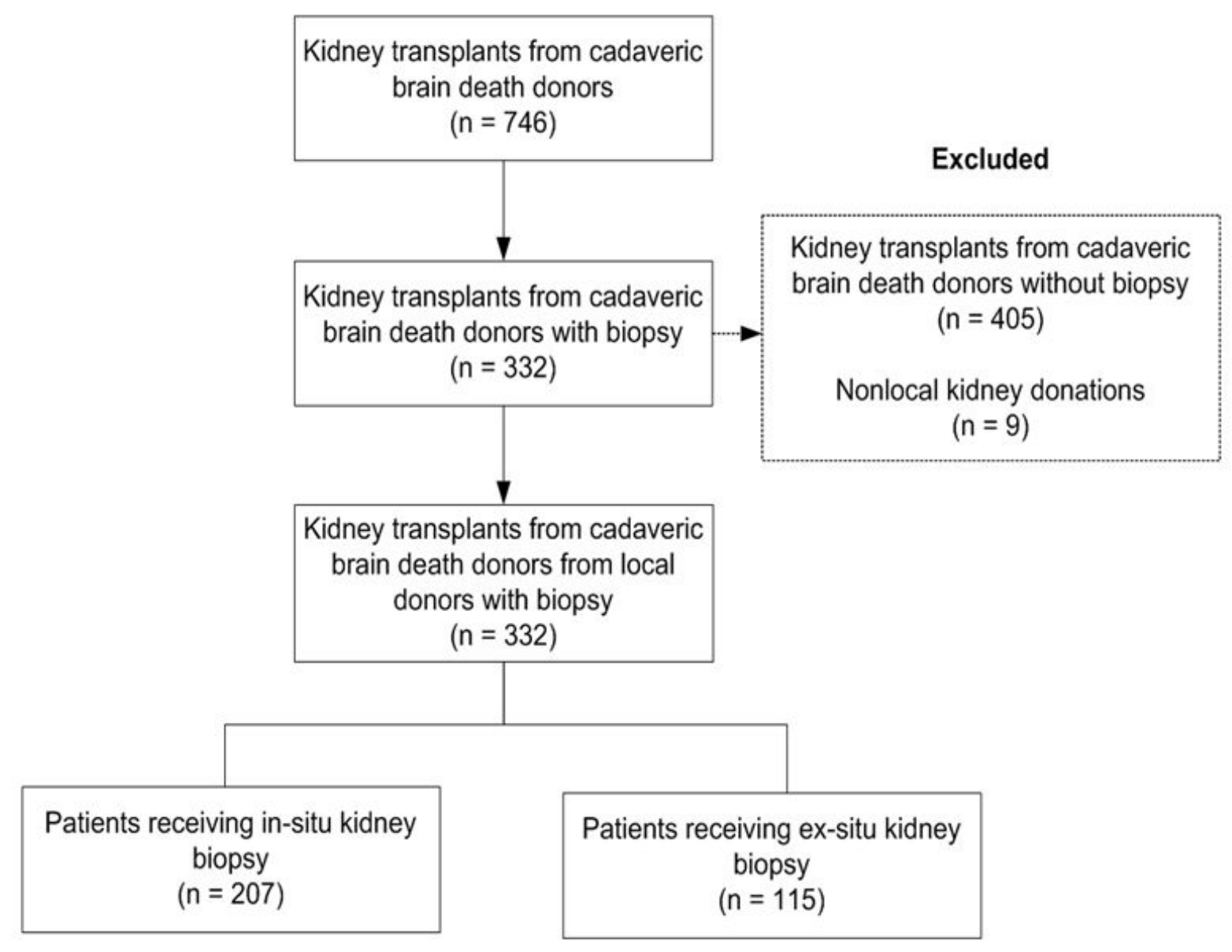

Figure 1

Flowchart of the study. 

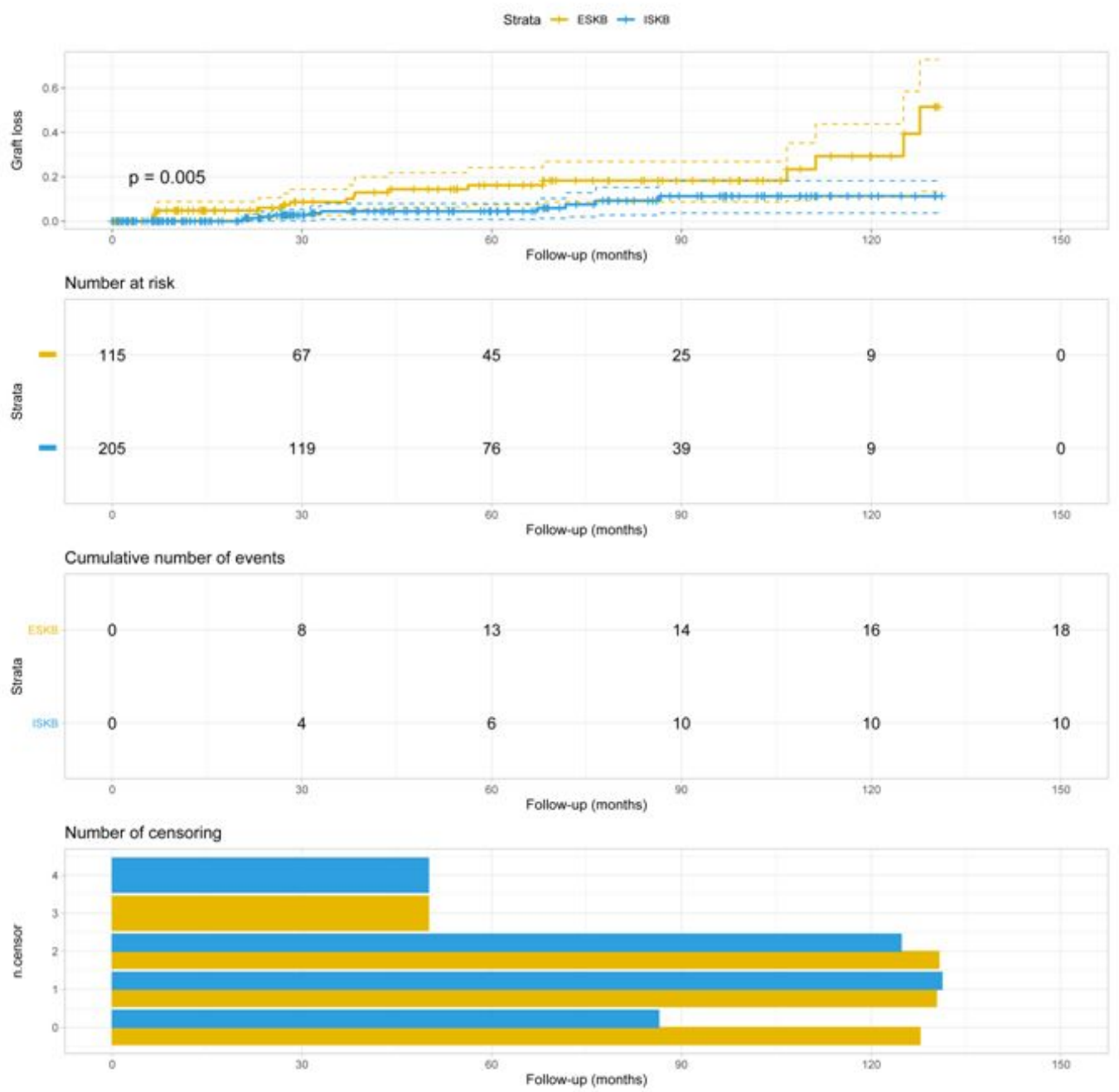

Figure 2

Cumulative incidence functions of graft loss for ESKB and ISKB cohorts. 


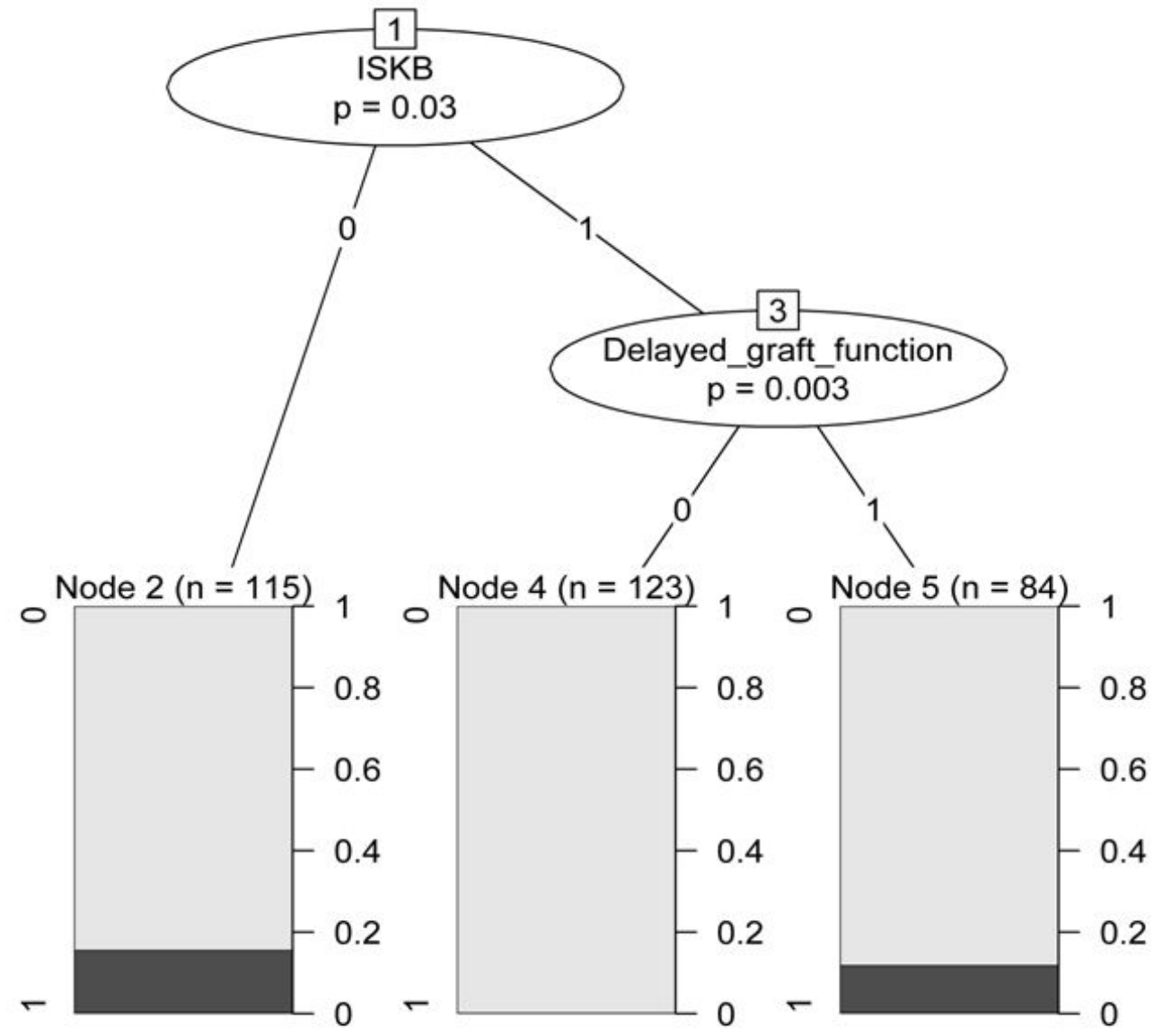

Figure 3

Conditional inference tree used to identify subgroups with increased risk of graft loss. ISKB $=1$ or rather ISKB $=$ yes. 

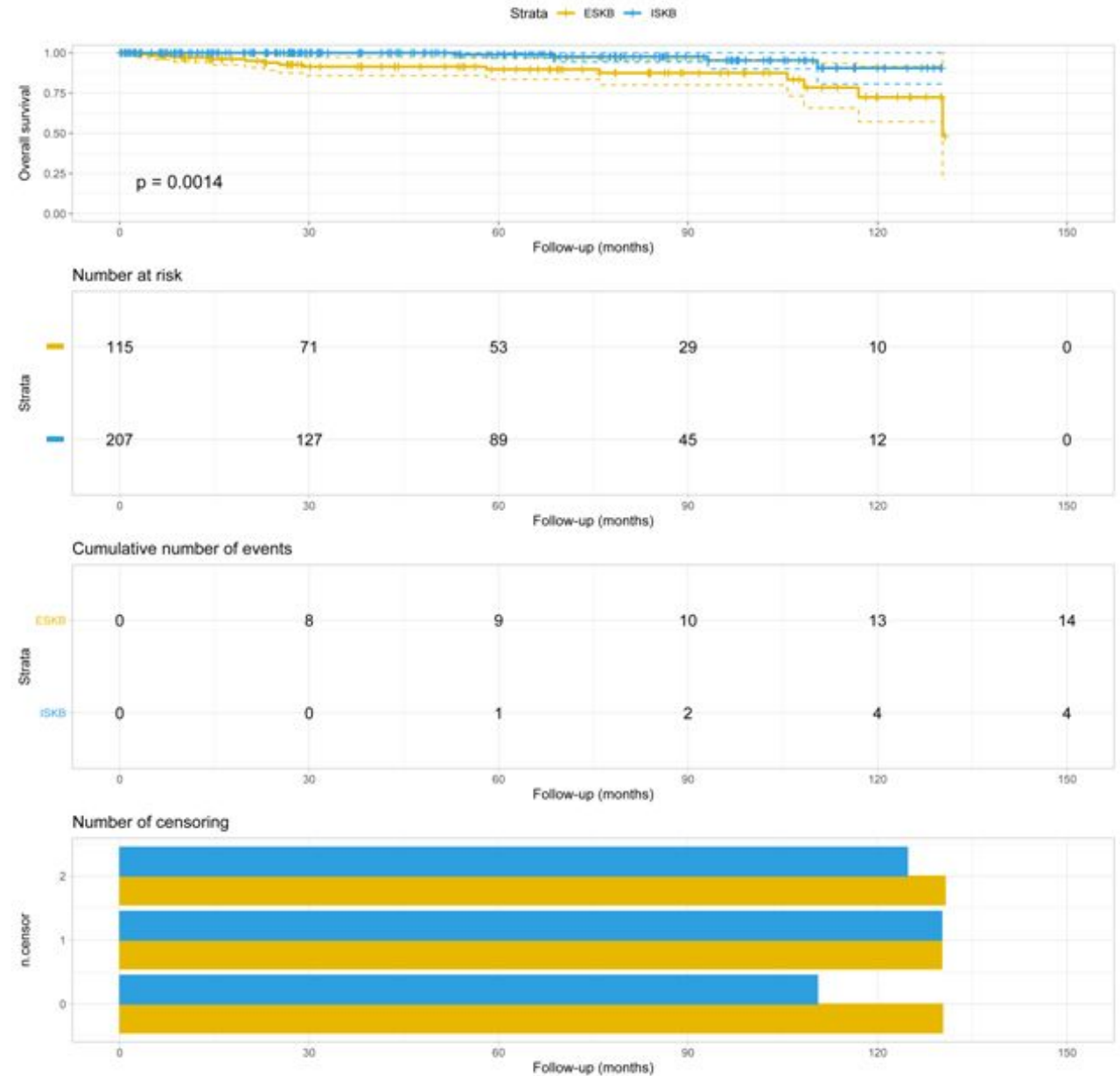

Figure 4

Overall survival of ESKB and ISKB cohorts. 